\title{
PLANE SETS ALLOWING BILIPSCHITZ EXTENSIONS
}

\author{
P. ALESTALO and D. A. TROTSENKO*
}

\begin{abstract}
We give a geometric characterization for a plane set $A \subset \mathrm{R}^{2}$ to have the following linear bilipschitz extension property: For $0 \leq \varepsilon \leq \delta$, every $(1+\varepsilon)$-bilipschitz map $f: A \rightarrow \mathrm{R}^{2}$ has a $(1+C \varepsilon)$ bilipschitz extension to the whole plane $\mathrm{R}^{2}$.
\end{abstract}

\section{Introduction}

Let $A$ be a subset of the Euclidean $n$-space $\mathbf{R}^{n}$ and let $L \geq 1$. A map $f: A \rightarrow \mathbf{R}^{n}$ is $L$-bilipschitz if

$$
|x-y| / L \leq|f(x)-f(y)| \leq L|x-y|
$$

for all $x, y \in A$.

In general, an $L$-bilipschitz map $f: A \rightarrow \mathrm{R}^{n}$ cannot be extended to a bilipschitz map $F: \mathrm{R}^{n} \rightarrow \mathrm{R}^{n}$, not even to a homeomorphism, but this is often possible in the case the bilipschitz constant $L$ is close to 1 . Extensions of this kind are interesting because of their connections with embeddings in Banach spaces and possible applications in theoretical computer science, cf. [4, p. 6] and [5, Ch. 15]. However, even in the Euclidean case there are few results that characterize the sets that have such extension properties. The main goal of this article is to give such a characterization for planar sets under the condition that an initial error term $\varepsilon$ is allowed to grow at most linearly to $C \varepsilon$. In order to understand this property in a more general context, we recall the following concepts.

Let $\Phi$ be the set of increasing homeomorphisms $\varphi:[0, \infty) \rightarrow[0, \infty)$. If $\varphi \in \Phi$ and $\delta>0$, we say that a set $A \subset \mathrm{R}^{n}$ has the $(\varphi, \delta)$-bilipschitz extension property, $(\varphi, \delta)$-BLEP for short, if for $0 \leq \varepsilon \leq \delta$, every $(1+\varepsilon)$-bilipschitz map $f: A \rightarrow \mathbf{R}^{n}$ has an extension to a $(1+\varphi(\varepsilon))$-bilipschitz map $F: \mathbf{R}^{n} \rightarrow \mathbf{R}^{n}$. We say that a set $A \subset \mathrm{R}^{n}$ belongs to the class $\varphi$-BLEP if it has the $(\varphi, \delta)$-BLEP for some $\delta>0$. In the case $\varphi(\varepsilon)=C \varepsilon$ we say that $A$ has the $(C, \delta)$-linear BLEP.

\footnotetext{
* This cooperation was supported by the Vilho, Yrjö and Kalle Väisälä Fund.
}

Received October 22, 2007; in revised form January 18, 2008. 
The 1-dimensional case is somewhat exceptional for the following reason. For $A \subset \mathrm{R}$, an embedding $f: A \rightarrow \mathrm{R}$ has a homeomorphic extension $F: \mathrm{R} \rightarrow$ $\mathrm{R}$ if and only if it is monotone. This result has a bilipschitz counterpart: a monotone $L$-bilipschitz map $f: A \rightarrow \mathrm{R}$ can be extended to an $L$-bilipschitz map $F: \mathrm{R} \rightarrow \mathrm{R}$ (with the same constant) by using a piecewise linear construction. Therefore, a set $A \subset \mathrm{R}$ has the $(1, \delta)$-linear BLEP if and only if $(1+\varepsilon)$-bilipschitz maps $f: A \rightarrow \mathbf{R}$ are monotone for $\varepsilon \leq \delta$. Thus all the $\varphi$-BLEP classes are the same in dimension one.

It was shown in [3] that a set $A \subset \mathrm{R}^{n}$ has $(C, \delta)$-linear BLEP if it satisfies a geometric condition called sturdiness; see 2.2 for the definition. In this article we prove that the converse is true in the 2-dimensional case. More precisely, we obtain the following theorem.

Theorem 1.1. Let $A \subset \mathrm{R}^{2}$ contain at least three points. Then the following assertions are quantitatively equivalent:

(1) A is c-sturdy.

(2) A has the $(C, \delta)$-linear BLEP.

Here quantitative equivalence means that $C$ and $\delta$ depend only on $c$, and conversely, $c=c(C, \delta)$.

The proof is given in subsection 4.3. Note that a set $A \subset \mathrm{R}^{n}$ consisting of at most two points has the 1-linear BLEP but it is sturdy only in the cases $n=1$ or $\# A=1$.

For extension problems in higher dimensions and with more general bounds for the bilipschitz constant, see [7] and the references in [3].

AcKNowledgements. We thank Antti Rasila for his help in drawing the figure in Section 3, as well as Jussi Väisälä and the referee for useful remarks and corrections concerning the whole manuscript.

\section{Basic concepts}

Our notation is standard and the same as in [3]. However, we recall the abbreviation $A(a, r)=A \cap \bar{B}(a, r)$ for a subset $A \subset \mathrm{R}^{n}$ and the following three geometric properties of sets that are needed in our main result.

2.1. Thickness. For each unit vector $e \in S^{n-1}$ we define the projection $\pi_{e}: \mathrm{R}^{n} \rightarrow \mathrm{R}$ by $\pi_{e} x=x \cdot e$. Let $A \neq \emptyset$ be a bounded set in $\mathrm{R}^{n}$. The thickness of $A$ is the number

$$
\theta(A)=\inf \left\{d\left(\pi_{e} A\right): e \in S^{n-1}\right\} .
$$


Alternatively, $\theta(A)$ is the infimum of all $t>0$ such that $A$ lies between two parallel hyperplanes $F, F^{\prime}$ with $d\left(F, F^{\prime}\right)=t$. We have always $0 \leq \theta(A) \leq$ $d(A)$.

2.2. Sturdiness. Let $A \subset \mathrm{R}^{n}$. For $a \in A$ we set $s(a)=s_{A}(a)=d(a, A \backslash$ $\{a\})$. Then $s(a)>0$ if and only if $a$ is isolated in $A$.

Let $c \geq 1$. We say that the set $A \subset \mathrm{R}^{n}$ is $c$-sturdy if

(1) $\theta(A(a, r)) \geq 2 r / c$ whenever $a \in A, r \geq c s(a), A \not \subset B(a, r)$,

(2) $\theta(A) \geq d(A) / c$.

If $A$ is unbounded, we omit (2), and the condition $A \not \subset B(a, r)$ of (1) is unnecessary.

Examples of sturdy sets in the plane include bounded Lipschitz-domains, $Z^{2}$, and the snowflake curve.

2.3. Relative connectivity $[6,4.6]$. Let $A \subset \mathrm{R}^{n}$ and $M \geq 1$. A sequence $\left(x_{0}, x_{1}, \ldots, x_{N-1}, x_{N}\right)$ is proper if $x_{j-1} \neq x_{j}$ for all $j$. A sequence $\left(x_{0}, x_{1}, \ldots\right.$, $\left.x_{N-1}, x_{N}\right)$ in $A$ is $M$-relative in $A$ if it is proper and

$$
\left|x_{j-1}-x_{j}\right| / M \leq\left|x_{j}-x_{j+1}\right| \leq M\left|x_{j-1}-x_{j}\right|
$$

for all $j$. Such a sequence is said to join the pairs $\left(x_{0}, x_{1}\right)$ and $\left(x_{N-1}, x_{N}\right)$. The set $A$ is $M$-relatively connected (abbr. RC) if every two proper pairs in $A$ can be joined by an $M$-relative sequence in $A$.

The simplest examples of relatively connected sets are the connected ones, but also many totally disconnected sets like the Cantor middle-third set satisfy the RC-condition.

Lemma 2.4. Let $A \subset \mathrm{R}^{n}$ be a closed c-sturdy set. Then $A$ is $c_{1}$-RC for every $c_{1}>c$.

Proof. Let $a \in A$ and $r>0$. Let $c_{1}>c$ and assume that $A \cap \bar{B}(a, r) \neq\{a\}$ and $A \not \subset \bar{B}(a, r)$. If $R(a, r)=\left\{x \in A\left|r / c_{1} \leq\right| x-a \mid \leq r\right\}=\emptyset$, then $\theta(A(a, r)) \leq \theta\left(\bar{B}\left(a, r / c_{1}\right)\right)=2 r / c_{1}<2 r / c$, a contradiction with the $c$ sturdiness of $A$. It follows that, under the above assumptions, $R(a, r) \neq \emptyset$, and by $[6,4.11]$, this implies the claim.

2.5. Linear isometric approximation property. Let $A \subset \mathbf{R}^{n}$. We say that $A$ has the $(C, \delta)$-linear isometric approximation property (IAP) if given $0<\varepsilon \leq$ $\delta$, a $(1+\varepsilon)$-bilipschitz map $f: A \rightarrow \mathrm{R}^{n}$, a point $a \in A$ and $r>0$, there is an isometry $T=T_{a, r}: \mathrm{R}^{n} \rightarrow \mathrm{R}^{n}$ such that

$$
|T x-f(x)| \leq C \varepsilon r
$$


for all $x \in A \cap \bar{B}(a, r)$.

Theorem 2.6. Suppose that a set $A \subset \mathrm{R}^{n}$ has the $(C, \delta)$-linear BLEP. Then it has the $\left(C_{1}, \delta\right)$-linear IAP with $C_{1}=C_{1}(C, n)$.

Proof. Let $f: A \rightarrow \mathrm{R}^{n}$ be $(1+\varepsilon)$-bilipschitz with $0<\varepsilon \leq \delta$. Suppose that $a \in A$ and $r>0$. Since $A$ has the $(C, \delta)$-linear BLEP, there is a $(1+C \varepsilon)$ bilipschitz extension $F: \mathrm{R}^{n} \rightarrow \mathrm{R}^{n}$ of $f$. Let $F_{a, r}=F \mid \bar{B}(a, r)$. Then $F_{a, r}$ is a $2 C \varepsilon r$-nearisometry and since $\theta(\bar{B}(a, r))=d(\bar{B}(a, r))$, [2,3.3] gives an isometry $T=T_{a, r}: \mathrm{R}^{n} \rightarrow \mathrm{R}^{n}$ such that

$$
\left\|T-F_{a, r}\right\|_{\bar{B}(a, r)} \leq 2 c_{n} C \varepsilon r .
$$

In particular, we have $|T x-f(x)| \leq 2 C c_{n} \varepsilon r$ for every $x \in A(a, r)$, and the proof is complete with $C_{1}=2 c_{n} C$.

\section{Triangle maps}

Since we work with the planar case, we use complex numbers whenever it simplifies notation.

3.1. Basic map. The basic triangle map $f:\{-1,0,1\} \rightarrow \mathbf{R}^{2}$ is defined by

$$
f( \pm 1)= \pm 1 \quad \text { and } \quad f(0)=i \sqrt{\varepsilon} .
$$

This map is $(1+\varepsilon)$-bilipschitz, but any approximation of $f$ by an isometry $T$ has an error at least $\sqrt{\varepsilon} / 2$. This is seen by minimizing the distance from the image of $f$ to the straight line $T \mathrm{R}$. The following elementary lemma generalizes this idea.

Lemma 3.2. Let $0 \leq \delta \leq \delta^{\prime} \leq 1 / 4$, let $A=\{-1, a, 1\} \subset \mathbf{R}^{2}$ be such that $\theta(A)=\left|a_{2}\right| \leq 2 \delta$, and let $f: A \rightarrow \mathbf{R}^{2}$ satisfy $f( \pm 1)= \pm 1$ and $\theta(f A)=$ $\left|f(a)_{2}\right| \geq 2 \delta^{\prime}$. If the disks $\bar{B}\left( \pm 1, \delta^{\prime}-\delta\right)$ and $\bar{B}\left(f(a), \delta^{\prime}+\delta\right)$ are disjoint, then every isometry $T: \mathbf{R}^{2} \rightarrow \mathbf{R}^{2}$ satisfies $\|T-f\|_{A} \geq \delta^{\prime}-\delta$.

Proof. We emphasize that the conditions $\theta(A)=\left|a_{2}\right|$ and $\theta(f A)=$ $\left|f(a)_{2}\right|$ belong to the assumpions. In particular, they imply that $-1<a_{1}<1$ and $-1<f(a)_{1}<1$ so that the situation is not too far from the basic map above.

Suppose that $T$ is an isometry with $\|T-f\|_{A}<\delta^{\prime}-\delta$ and let $L=T \mathrm{R}$. Writing $a^{\prime}=\left(a_{1}, 0\right)$, we have

$$
\left|T a^{\prime}-T a\right|=\left|a^{\prime}-a\right|=\left|a_{2}\right| \leq 2 \delta .
$$

If $L$ does not meet the disk $B\left(f(a), \delta^{\prime}+\delta\right)$, then

$$
|T a-f(a)| \geq\left|T a^{\prime}-f(a)\right|-\left|T a^{\prime}-T a\right| \geq\left(\delta^{\prime}+\delta\right)-2 \delta=\delta^{\prime}-\delta,
$$


a contradiction.

It follows that the line $L$ meets all three disks $\bar{B}\left( \pm 1, \delta^{\prime}-\delta\right)$ and $B\left(f(a), \delta^{\prime}+\right.$ $\delta)$. By assumption, these disks are disjoint, and by elementary geometry we get

$$
\left(\delta^{\prime}-\delta\right)+\left(\delta^{\prime}+\delta\right)>\left|f(a)_{2}\right|=\theta(f A) \geq 2 \delta^{\prime},
$$

which leads to a contradiction. The result follows from this.

Later on we will need maps that are defined on a narrow neighbourhood of a line but that still possess the essential features of the basic triangle map: they should be $(1+c \varepsilon)$-bilipschitz but their approximation by isometries should produce an error of the order $\sqrt{\varepsilon}$. The following lemmas show how to construct these maps.

Lemma 3.3. Let $0 \leq \varepsilon \leq 1 / 10$ and let $a, b \in[0,1]$ be such that $2 \varepsilon \leq a \leq$ $b / 2$. Then there is a $C^{2}$ function $f: \mathbf{R} \rightarrow \mathbf{R}$ satisfying

(i) $f(x)=0$ for $x \leq 0$ and $x \geq b$;

(ii) $f(a)=\varepsilon^{3 / 2}$;

(iii) $f$ is $2 \sqrt{\varepsilon}$-Lipschitz;

(iv) the curvature $K$ of the graph $y=f(x)$ satisfies $K \leq 1 / \sqrt{\varepsilon}$.

Proof. Let $0<o<a$ and consider first the interval $[o, a]$. One should think that $o \approx 0$, but we need $o>0$ for technical reasons. Let $r=\sqrt{\varepsilon}$. The graph $y=f(x)$ consists of two circular arcs and a line segment. The construction is based on the diagram below, where also the notation is indicated.

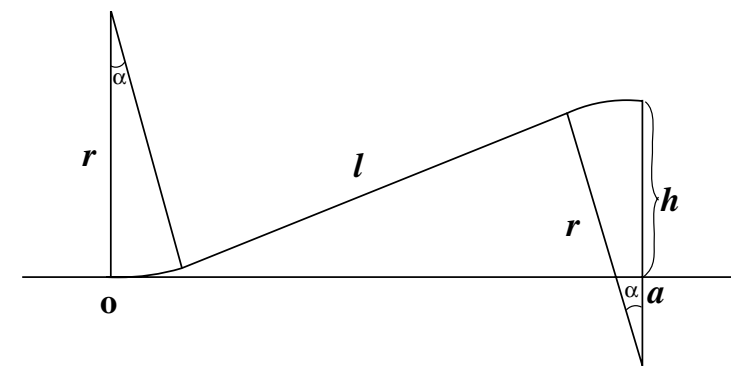

Part of the graph $y=f(x)$ with $h=\varepsilon \sqrt{\varepsilon}$.

By elementary geometry the variables $l$ and $\alpha$ must satisfy

$$
\left\{\begin{array}{l}
2 r \sin \alpha+l \cos \alpha=a-o \\
2 r(1-\cos \alpha)+l \sin \alpha=\varepsilon^{3 / 2},
\end{array}\right.
$$


and this system has the exact solution

$$
\begin{aligned}
l & =\sqrt{(a-o)^{2}-4 \varepsilon^{2}+\varepsilon^{3}}, \\
\alpha & =\arcsin \left(\sqrt{\varepsilon}(2(a-o)+l \varepsilon-2 l) /\left(l^{2}+4 \varepsilon\right)\right) .
\end{aligned}
$$

The Lipschitz condition requires that $\tan \alpha \leq 2 \sqrt{\varepsilon}$. It is geometrically obvious that $\alpha$ is decreasing in $a$, and thus $\alpha$ attains its maximum at $a=2 \varepsilon$. By substituting this value and choosing $o$ small enough, we obtain $\alpha \leq \arcsin \sqrt{\varepsilon} \leq$ $\arctan (2 \sqrt{\varepsilon})$.

A similar construction is used on the interval $[a, b]$, and outside $[o, b-o]$ we define $f(x)=0$. This function satisfies conditions (i)-(iv), but it is only piecewise $C^{2}$. However, at the six points where a circular arc is joined either to another arc or to a line segment, we use standard smoothing by clothoids (aka Cornu spirals), in an arbitrarily small neighbourhood of each joint, in such a way that the Lipschitz constant does not change, the curvature stays between the appropriate bounds, and the support of $f$ does not expand outside $[0, b]$; see $[1$, p. 636] for the basic construction.

Using the following lemma we can construct tubular neighbourhood extensions for mappings of the type $x \mapsto(x, f(x))$.

Lemma 3.4. Let $0<\varepsilon<1 / 10$, let $I \subset \mathbf{R}$ be an interval and let $f: I \rightarrow \mathbf{R}$ be $\sqrt{\varepsilon}$-Lipschitz and $C^{2}$. Define $F: I \times[-\delta, \delta] \rightarrow \mathrm{R}^{2}$ by setting

$$
F(x, y)=x+i f(x)+y \mathbf{n}(x)
$$

where $\mathbf{n}(x)$ is the upper unit normal to the graph $y=f(x)$. Let $K$ be the maximal curvature of $y=f(x)$. If $K \delta \leq \varepsilon$, then $F$ is $(1+4 \varepsilon)$-bilipschitz. Moreover, if $f(x)=0$ except for a subinterval of length $l$, then $|F(z)-z| \leq$ $\sqrt{\varepsilon} l+\delta$ for every $z \in I \times[-\delta, \delta]$.

Proof. Let $z_{i}=\left(x_{i}, y_{i}\right) \in I \times[-\delta, \delta], i=1,2$. Note that

$$
|y| \leq \delta, \quad\left|f^{\prime}(x)\right| \leq \sqrt{\varepsilon} \quad \text { and } \quad \frac{\left|f^{\prime \prime}(x)\right|}{\left(1+f^{\prime}(x)^{2}\right)^{3 / 2}} \leq K
$$

for all $(x, y)$.

In complex form we have

$$
\mathbf{n}(x)=\frac{1}{\sqrt{1+f^{\prime}(x)^{2}}}\left(-f^{\prime}(x)+i\right) .
$$


Thus

$$
\begin{aligned}
\mid F\left(z_{1}\right)- & \left.F\left(z_{2}\right)\right|^{2} \\
= & \left|x_{1}-x_{2}\right|^{2}+\left|\frac{y_{1} f^{\prime}\left(x_{1}\right)}{\sqrt{1+f^{\prime}\left(x_{1}\right)^{2}}}-\frac{y_{2} f^{\prime}\left(x_{2}\right)}{\sqrt{1+f^{\prime}\left(x_{2}\right)^{2}}}\right|^{2} \\
& \left|f\left(x_{1}\right)-f\left(x_{2}\right)\right|^{2}+\left|\frac{y_{1}}{\sqrt{1+f^{\prime}\left(x_{1}\right)^{2}}}-\frac{y_{2}}{\sqrt{1+f^{\prime}\left(x_{2}\right)^{2}}}\right|^{2} \\
& -2\left(x_{1}-x_{2}\right)\left(\frac{y_{1} f^{\prime}\left(x_{1}\right)}{\sqrt{1+f^{\prime}\left(x_{1}\right)^{2}}}-\frac{y_{2} f^{\prime}\left(x_{2}\right)}{\sqrt{1+f^{\prime}\left(x_{2}\right)^{2}}}\right) \\
& +2\left(f\left(x_{1}\right)-f\left(x_{2}\right)\right)\left(\frac{y_{1}}{\sqrt{1+f^{\prime}\left(x_{1}\right)^{2}}}-\frac{y_{2}}{\sqrt{1+f^{\prime}\left(x_{2}\right)^{2}}}\right) .
\end{aligned}
$$

Writing the right hand side above as $\left|x_{1}-x_{2}\right|^{2}+t_{1}+t_{2}+t_{3}+t_{4}$, where $t_{4}$ contains the last two terms, we have to estimate each term. Since $F$ is defined in a convex set, we can use the mean value theorem.

(i) To estimate $t_{1}$, let $g(x, y)=y f^{\prime}(x) / \sqrt{1+f^{\prime}(x)^{2}}$. Then

$$
|\nabla g|^{2}=\frac{y^{2} f^{\prime \prime}(x)^{2}}{\left(1+f^{\prime}(x)^{2}\right)^{3}}+\frac{f^{\prime}(x)^{2}}{1+f^{\prime}(x)^{2}} \leq \delta^{2} K^{2}+\varepsilon \leq 2 \varepsilon,
$$

which implies that $t_{1} \leq 2 \varepsilon\left|z_{1}-z_{2}\right|^{2}$.

(ii) The upper bound $t_{2} \leq \varepsilon\left|x_{1}-x_{2}\right|^{2}$ follows from the Lipschitz condition.

(iii) We need both upper and lower bounds for $t_{3}$. Applying the mean value theorem for $h(x, y)=y / \sqrt{1+f^{\prime}(x)^{2}}$, we get

$$
t_{3}=\left(-\frac{f^{\prime}(u) f^{\prime \prime}(u) v}{\left(1+f^{\prime}(u)^{2}\right)^{3 / 2}}\left(x_{1}-x_{2}\right)+\frac{1}{\sqrt{1+f^{\prime}(u)^{2}}}\left(y_{1}-y_{2}\right)\right)^{2}
$$

where $(u, v)$ lies on the segment $\left[z_{1}, z_{2}\right]$. Using the estimate $2 \varepsilon^{3 / 2}\left|x_{1}-x_{2}\right|\left|y_{1}-y_{2}\right| \leq 2 \varepsilon\left|x_{1}-x_{2}\right|\left|y_{1}-y_{2}\right| \leq \varepsilon\left|x_{1}-x_{2}\right|^{2}+\varepsilon\left|y_{1}-y_{2}\right|^{2}$, it follows that

$$
\begin{aligned}
t_{3} & \leq \varepsilon K^{2} \delta^{2}\left|x_{1}-x_{2}\right|^{2}+\frac{1}{1+f^{\prime}(u)^{2}}\left|y_{1}-y_{2}\right|^{2}+2 \sqrt{\varepsilon} K \delta\left|x_{1}-x_{2}\right|\left|y_{1}-y_{2}\right| \\
& \leq \varepsilon^{3}\left|x_{1}-x_{2}\right|^{2}+\left|y_{1}-y_{2}\right|^{2}+\varepsilon\left|x_{1}-x_{2}\right|^{2}+\varepsilon\left|y_{1}-y_{2}\right|^{2} \\
& \leq 2 \varepsilon\left|x_{1}-x_{2}\right|^{2}+(1+\varepsilon)\left|y_{1}-y_{2}\right|^{2} .
\end{aligned}
$$


In the opposite direction, we have

$$
\begin{aligned}
t_{3} & \geq \frac{1}{1+\varepsilon}\left|y_{1}-y_{2}\right|^{2}-2 \sqrt{\varepsilon} K \delta\left|x_{1}-x_{2}\right|\left|y_{1}-y_{2}\right| \\
& \geq(1-2 \varepsilon)\left|y_{1}-y_{2}\right|^{2}-\varepsilon\left|x_{1}-x_{2}\right|^{2} .
\end{aligned}
$$

(iv) Rearranging and using the Taylor formula, we have

$$
\begin{aligned}
t_{4}= & \frac{2 y_{1}}{\sqrt{1+f^{\prime}\left(x_{1}\right)^{2}}}\left(f\left(x_{1}\right)-f\left(x_{2}\right)-f^{\prime}\left(x_{1}\right)\left(x_{1}-x_{2}\right)\right) \\
& +\frac{2 y_{2}}{\sqrt{1+f^{\prime}\left(x_{2}\right)^{2}}}\left(f^{\prime}\left(x_{2}\right)\left(x_{1}-x_{2}\right)-f\left(x_{1}\right)+f\left(x_{2}\right)\right) \\
= & \left(\frac{y_{1} f^{\prime \prime}\left(\xi_{1}\right)}{\sqrt{1+f^{\prime}\left(x_{1}\right)^{2}}}-\frac{y_{2} f^{\prime \prime}\left(\xi_{2}\right)}{\sqrt{1+f^{\prime}\left(x_{2}\right)^{2}}}\right)\left|x_{1}-x_{2}\right|^{2},
\end{aligned}
$$

where $\xi_{1}, \xi_{2} \in\left[x_{1}, x_{2}\right]$. Since $\left|f^{\prime \prime}(\xi)\right| \leq K(1+\varepsilon)^{3 / 2}$, this implies that

$$
\left|t_{4}\right| \leq 2 K \delta(1+\varepsilon)^{3 / 2}\left|x_{1}-x_{2}\right|^{2} \leq 3 \varepsilon\left|x_{1}-x_{2}\right|^{2} .
$$

Using these estimates we obtain

$$
\begin{aligned}
\left|F\left(z_{1}\right)-F\left(z_{2}\right)\right|^{2} \leq & \left|x_{1}-x_{2}\right|^{2}+2 \varepsilon\left|x_{1}-x_{2}\right|^{2}+2 \varepsilon\left|y_{1}-y_{2}\right|^{2}+\varepsilon\left|x_{1}-x_{2}\right|^{2} \\
& +2 \varepsilon\left|x_{1}-x_{2}\right|^{2}+(1+\varepsilon)\left|y_{1}-y_{2}\right|^{2}+3 \varepsilon\left|x_{1}-x_{2}\right|^{2} \\
= & (1+8 \varepsilon)\left|x_{1}-x_{2}\right|^{2}+(1+3 \varepsilon)\left|y_{1}-y_{2}\right|^{2}
\end{aligned}
$$

so that $\left|F\left(z_{1}\right)-F\left(z_{2}\right)\right| \leq \sqrt{1+8 \varepsilon}\left|z_{1}-z_{2}\right| \leq(1+4 \varepsilon)\left|z_{1}-z_{2}\right|$.

For the lower bound, we discard irrelevant positive terms and get

$$
\begin{aligned}
\left|F\left(z_{1}\right)-F\left(z_{2}\right)\right|^{2} & \geq\left|x_{1}-x_{2}\right|^{2}+t_{3}-\left|t_{4}\right| \\
& \geq(1-4 \varepsilon)\left|x_{1}-x_{2}\right|^{2}+(1-2 \varepsilon)\left|y_{1}-y_{2}\right|^{2} \\
& \geq(1-4 \varepsilon)\left|z_{1}-z_{2}\right|^{2} .
\end{aligned}
$$

This implies that $\left|F\left(z_{1}\right)-F\left(z_{2}\right)\right| \geq \sqrt{1-4 \varepsilon}\left|z_{1}-z_{2}\right| \geq\left|z_{1}-z_{2}\right| /(1+4 \varepsilon)$.

The proof for the bilipschitz condition is now complete, and the last inequality is obvious.

Lemma 3.5. Let $A \subset \mathrm{R}^{n}$ and let $\varepsilon \leq 1 / 10$. Suppose that $a \in A, r>0$ and let $f: A \rightarrow \mathbf{R}^{n}$ be $(1+\varepsilon)$-bilipschitz such that $|f(z)-z| \leq \varepsilon r$ whenever $|z-a| \leq r / 2$ and $f(z)=z$ for $|z-a| \geq r / 2$. Define $F: A \cup\left(\mathrm{R}^{n} \backslash B(a, r)\right) \rightarrow \mathrm{R}^{n}$ by setting

$$
F(z)= \begin{cases}f(z) & \text { for } z \in A, \\ z & \text { for }|z-a| \geq r .\end{cases}
$$


Then $F$ is $(1+3 \varepsilon)$-bilipschitz.

Proof. Let $z_{1} \in A \cap B(a, r / 2)$ and $\left|z_{2}-a\right| \geq r$. Then $\left|z_{1}-z_{2}\right| \geq r / 2$, which implies that

$$
\begin{aligned}
\left|F\left(z_{1}\right)-F\left(z_{2}\right)\right|=\left|f\left(z_{1}\right)-z_{2}\right| & \leq\left|f\left(z_{1}\right)-z_{1}\right|+\left|z_{1}-z_{2}\right| \leq \varepsilon r+\left|z_{1}-z_{2}\right| \\
& \leq(1+2 \varepsilon)\left|z_{1}-z_{2}\right| .
\end{aligned}
$$

In the opposite direction, we have

$$
\begin{aligned}
\left|F\left(z_{1}\right)-F\left(z_{2}\right)\right|=\left|f\left(z_{1}\right)-z_{2}\right| & \geq\left|z_{1}-z_{2}\right|-\left|f\left(z_{1}\right)-z_{1}\right| \geq\left|z_{1}-z_{2}\right|-\varepsilon r \\
& \geq(1-2 \varepsilon)\left|z_{1}-z_{2}\right| \geq\left|z_{1}-z_{2}\right| /(1+3 \varepsilon),
\end{aligned}
$$

since $\varepsilon \leq 1 / 10$.

All other cases for $z_{1}, z_{2}$ are trivial, and the proof is complete.

Finally, we need an estimate on the distortion of angles under bilipschitz maps.

Lemma 3.6. Let $1<t \leq 2$ and let $f:\{0,1, t\} \rightarrow \mathrm{R}^{n}$ be $(1+\varepsilon)$-bilipschitz with $\varepsilon \leq 1 / 100$. Let $A=f(0), B=f(1), C=f(t)$ and $\alpha=\angle B A C$. Then $\alpha \leq 2.1 \sqrt{\varepsilon}$.

Proof. Consider the triangle with vertices $A, B, C$. By elementary geometry $\alpha$ is maximal in the case $A B=1+\varepsilon, B C=(t-1)(1+\varepsilon)$, and $A C=t /(1+\varepsilon)$. Using trigonometry and Taylor approximation we obtain

$$
\sin \alpha \leq 2 \sqrt{(t-1) \varepsilon} \leq 2 \sqrt{\varepsilon} \leq 0.2 .
$$

Furthermore, for these values we have $\alpha \leq 1.01 \sin \alpha \leq 2.1 \sqrt{\varepsilon}$, and the proof is complete.

\section{Main proofs}

We use triangle maps to prove the following theorem, which constitutes the first part of our main result.

Theorem 4.1. Let $\lambda \geq 1, c>(14 \lambda)^{8}$, and let $A \subset \mathbf{R}^{2}$ be $\lambda$-relatively connected but not c-sturdy. Then for $1 / \sqrt{c} \leq \varepsilon \leq 1 /(14 \lambda)^{4}$ there is a $(1+48 \varepsilon)$ $B L$ map $f: A \rightarrow \mathbf{R}^{2}$ with the following property: there are $a \in A$ and $r>0$ such that

$$
\|T-f\|_{A(a, r)} \geq \frac{r}{1000 \lambda^{3}} \sqrt{\varepsilon}
$$

for all isometries $T: \mathrm{R}^{2} \rightarrow \mathrm{R}^{2}$. 
Proof. Since $A$ is not $\left(1 / \varepsilon^{2}\right)$-sturdy, there are two possibilities.

Case 1: Condition 2.2(1) is not satisfied. In this case there are $a \in A$ and $r>0$ such that $A \not \subset B(a, r), s(a) \leq \varepsilon^{2} r$ and $\theta(A(a, r)) \leq 2 \varepsilon^{2} r$. By scaling, we may assume that $a=0, r=1$, and then $A \not \subset B(1)=B(0,1), s(0) \leq \varepsilon^{2}$, $\theta(A(0,1)) \leq 2 \varepsilon^{2}$. Furthermore, we may assume that $A(0,1)$ is contained in the $2 \varepsilon^{2}$-neighbourhood of $\mathbf{R} \subset \mathbf{R}^{2}$.

We apply [6, 4.11(2)] with $c=4 \lambda$ to find points $u, v \in A$ as follows. Since $s(0) \leq \varepsilon^{2}<\varepsilon$, the set $A(0,2.25 \varepsilon)$ contains at least two points. Also $A \not \subset B(1)$, and thus there is a point $u \in A \cap B(9 \lambda \varepsilon) \backslash B(2.25 \varepsilon)$. Similarly, since $80 \lambda^{2} \varepsilon \leq 1$, there is $v \in A \cap B\left(80 \lambda^{2} \varepsilon\right) \backslash B(20 \lambda \varepsilon)$. There are six possibilities for the order of the points $0, u_{1}, v_{1}$ and of these only two are essentially different; we consider the case where $0<u_{1}<v_{1}<1$, the other cases being similar. However, the constants appearing below apply for all cases and may thus seem unnecessarily large for this special case.

We construct a bilipschitz map $f: A \rightarrow \mathrm{R}^{2}$ as follows:

- Apply Lemma 3.3 with substitutions $0 \mapsto 0, a \mapsto u_{1}, b \mapsto v_{1}$, relying on the estimates $v_{1}>19 \lambda \varepsilon>2 u_{1}$ and $u_{1}>2 \varepsilon$. This gives a $2 \sqrt{\varepsilon}$-Lipschitz map $f_{1}: \mathbf{R} \rightarrow \mathbf{R}$ such that $f_{1}(x)=0$ if $x \notin\left[0, v_{1}\right], f_{1}\left(u_{1}\right)=\varepsilon^{3 / 2}$, and $K \leq 1 / \sqrt{\varepsilon}$.

- Apply Lemma 3.4 with $\varepsilon \mapsto(2 \sqrt{\varepsilon})^{2}=4 \varepsilon, \delta \mapsto 2 \varepsilon^{2}, I \mapsto \mathrm{R}$ and $f \mapsto f_{1}$. Then $K \delta \leq 2 \varepsilon^{3 / 2} \leq 4 \varepsilon$, and the resulting map $F: \mathrm{R} \times[-\delta, \delta] \rightarrow \mathrm{R}^{2}$ is $(1+16 \varepsilon)$-BL. Also, we have $l \leq 90 \lambda^{2} \varepsilon$ and therefore

$$
|F(z)-z| \leq 90 \lambda^{2} \varepsilon \sqrt{4 \varepsilon}+2 \varepsilon^{2}<\varepsilon
$$

for all $z$. This is the crucial estimate that determines the upper bound for $\varepsilon$.

- We extend the definition of $F$ outside $B(1)$ by $F(z)=z$. Substitute $\varepsilon \mapsto$ $16 \varepsilon$ and $r=1 / 2$ in Lemma 3.5. Since $90 \lambda^{2} \varepsilon \leq r / 2$, we have $|F(z)-z| \leq$ $\varepsilon \leq 16 \varepsilon r$ for $|z| \leq r / 2$ and $F(z)=z$ for $|z| \geq r / 2$. It follows that $F$ is $(1+48 \varepsilon)$-BL.

- The domain of definition for $F$ contains the set $A$ and by restriction we get the required $(1+48 \varepsilon)$-BL map $f: A \rightarrow \mathrm{R}^{2}$.

It remains to show that $f$ cannot be well approximated by isometries. For this it suffices to consider the restriction $f \mid\{0, u, v\}$ in the disk $B=\bar{B}\left(0, r_{1}\right)$, where $r_{1}=90 \lambda^{2} \varepsilon$. Let $A^{\prime}=\{0, u, v\}$ and let $h: \mathbf{R}^{2} \rightarrow \mathrm{R}^{2}$ be a similarity such that $h(0)=-1, h(v)=1$ and let $g=h f h^{-1}: h A^{\prime} \rightarrow h f A^{\prime}$. Since $f(0)=0, f(v)=v$, Lemma 3.2 can be applied to $g$. The similarity ratio $t$ of $h$ satisfies $1 / 45 \lambda^{2} \varepsilon \leq t \leq 1 / 10 \lambda \varepsilon$, and thus $\theta\left(h A^{\prime}\right) \leq 2 \varepsilon^{2} / 10 \lambda \varepsilon=\varepsilon / 5 \lambda$ and $\left.\theta\left(g h A^{\prime}\right)\right) \geq\left(\varepsilon^{3 / 2}-4 \varepsilon^{2}\right) / 45 \lambda^{2} \varepsilon>\sqrt{\varepsilon} / 46 \lambda^{2}$. Thus the error of approximation 
of $g$ by an isometry is at least

$$
\sqrt{\varepsilon} / 92 \lambda^{2}-\varepsilon / 10 \lambda \geq \sqrt{\varepsilon} / 100 \lambda^{2},
$$

and therefore

$$
\|T-f\|_{A\left(0, r_{1}\right)} \geq 10 \lambda \varepsilon\left(\sqrt{\varepsilon} / 100 \lambda^{2}\right)=\varepsilon^{3 / 2} / 10 \lambda>\frac{r_{1}}{1000 \lambda^{3}} \sqrt{\varepsilon}
$$

for all isometries $T$. This completes the proof for Case 1 .

Case 2: Condition 2.2(2) is not satisfied. This implies that $A$ is bounded and $\theta(A)<\varepsilon^{2} d(A)$. Using $\lambda$-relative connectedness, we can find points $a, b, c \in$ $A$ such that $1 \leq|a-b| /|b-c| \leq \lambda$. Using Lemmas 3.3 and 3.4, we can construct a map $f: A \rightarrow \mathbf{R}^{2}$ that by 3.2 contradicts the requirements. The details are similar to Case 1 and are omitted.

This completes the proof.

THEOREM 4.2. Let $\lambda \geq 1000$, let $A \subset \mathrm{R}^{n}$ be a closed set that is not $\lambda$ relatively connected. Then there is $\varepsilon \leq 2 /(\lambda-2)$ and a $(1+\varepsilon)$-bilipschitz map $f: A \rightarrow \mathrm{R}^{n}$ with the following property: If $F: \mathbf{R}^{n} \rightarrow \mathrm{R}^{n}$ is a $(1+\delta)$ bilipschitz extension of $f$, then

$$
\delta \geq 1 / 20 \ln ^{2} \varepsilon
$$

Proof. We use the concept of upper sets from $[6,4.9]$. Since $A$ is not $\lambda$ relatively connected, the upper set $\tilde{A}$ consists of more than one $\ln \lambda$-component. Let $\gamma$ be a $\ln \lambda$-component that is not the greatest element; see $[6,3.2]$. By $[6,3.4(11)$ and 3.4(14)] the set $\pi \gamma$ is compact, and by [6, 3.4(12)] we have $A \cap B(\pi \gamma,(\lambda-1) d(\pi \gamma))=\pi \gamma$. Choose $a, b \in \pi \gamma$ such that $|a-b|=d(\pi \gamma)$ and then $z \in A \backslash \pi \gamma$ such that $d(z, \pi \gamma)$ is minimal. We may assume that $|b-z| \leq|a-z|$, and hence $\angle a b z \geq \pi / 3$. Using suitable similarities, we may assume that $b=0,|a-b|=1$ and $z=t e_{1}$ with $t \geq \lambda-1$.

We choose $\varepsilon=2 /(t-1) \leq 2 /(\lambda-2)<0.01$ and construct a $(1+\varepsilon)$ bilipschitz map $f: A \rightarrow \mathrm{R}^{n}$ as follows. Let $f \mid(A \backslash B(0,1))=\mathrm{id}$, and let $f$ rotate $\bar{B}(0,1)$ so that $f(0)=0$ and $f(a)=e_{1}$. To calculate the bilipschitz constant $L$ of $f$, we note that the worst case arises from $a=-e_{1}, f(a)=e_{1}$; this seems geometrically obvious and can be proved by solving an elementary extremal value problem. Thus

$$
L \leq \frac{t+1}{t-1}=1+\frac{2}{t-1}=1+\varepsilon .
$$

Suppose now that $f$ can be extended to a $(1+\delta)$-bilipschitz map $F: \mathbf{R}^{n} \rightarrow$ $\mathrm{R}^{n}$. We apply Lemma 3.6 to the map $F^{-1} \mid\left\{0, e_{1}, 2 e_{1}, 4 e_{1}, \ldots, 2^{N} e_{1}, z\right\}$, 
where $N=\left\lfloor\log _{2} t\right\rfloor$. Let $a_{i}=F^{-1}\left(2^{i} e_{1}\right)$ for $i=0,1,2, \ldots, N$ and $a_{N+1}=z$. The lemma implies that $\angle a_{i} 0 a_{i+1} \leq 2.1 \sqrt{\delta}$, and therefore

$$
\begin{aligned}
1 \leq \frac{\pi}{3} \leq \angle a 0 z \leq \sum_{i=0}^{N} \angle a_{i} 0 a_{i+1} & \leq 2.1 \sqrt{\delta}(N+1) \leq 2.1 \sqrt{\delta}\left(\log _{2} t+1\right) \\
& \leq 2.1 \sqrt{\delta}(1.5 \ln t+1) \leq 3.15 \sqrt{\delta} \ln (2 t) .
\end{aligned}
$$

Since $t=2 / \varepsilon+1 \leq 2.1 / \varepsilon$, we obtain

$$
\delta \geq \frac{1}{10 \ln ^{2}(4.2 / \varepsilon)} \geq \frac{1}{20 \ln ^{2} \varepsilon} .
$$

This completes the proof.

4.3. Proof of Theorem 1.1. The implication $(1) \Rightarrow(2)$ was the main result of [3].

For the converse part, suppose that $A$ has the $(C, \delta)$-linear BLEP. Choose $s_{0}=s_{0}(C)>0$ such that $g(s)=20 C s \ln ^{2} s<1$ for $0<s \leq s_{0}$ and set $\lambda=\lambda(C, \delta)=\max \left\{1000,2+2 /\left(\delta \wedge s_{0}\right)\right\}$.

We first show that $A$ is $\lambda$-RC. If this is not the case, then Theorem 4.2 gives an $\varepsilon \leq 2 /(\lambda-2)$ and a $(1+\varepsilon)$-bilipschitz map $f: A \rightarrow \mathrm{R}^{2}$. As $\varepsilon \leq \delta$, the $(C, \delta)$-linear BLEP of $A$ gives a $(1+C \varepsilon)$-bilipschitz extension $F: \mathrm{R}^{2} \rightarrow \mathrm{R}^{2}$ of $f$. By 4.2 we have $g(\varepsilon) \geq 1$, which gives the contradiction $\varepsilon>s_{0} \geq 2 /(\lambda-2)$ and proves that $A$ is $\lambda$-RC.

To prove that $A$ is $c_{0}$-sturdy with $c_{0}(C, \delta)$, we assume that $A$ is not $c$-sturdy for some $c>(14 \lambda)^{8} \vee 48^{2} / \delta^{2}$. Writing $\varepsilon_{1}=1 / \sqrt{c}$, we have $\varepsilon_{1}<1 /(14 \lambda)^{4}$. Hence 4.1 gives a $\left(1+48 \varepsilon_{1}\right)$-bilipschitz map $f_{1}: A \rightarrow \mathrm{R}^{2}$, a point $a \in A$ and a radius $r>0$ such that

$$
\left\|T-f_{1}\right\|_{A(a, r)} \geq r \sqrt{\varepsilon_{1}} / 1000 \lambda^{3}
$$

for every isometry $T$ of $\mathrm{R}^{2}$.

By Theorem 2.6 the set $A$ has the $\left(C_{1}, \delta\right)$-IAP with $C_{1}(C)$. As $48 \varepsilon_{1} \leq \delta$, there is an isometry $T_{1}$ of $\mathrm{R}^{2}$ such that $\left\|T_{1}-f_{1}\right\| \leq 48 C_{1} \varepsilon_{1} r$, which implies that

$$
c=1 / \varepsilon_{1}^{2} \leq\left(48 \cdot 1000 C_{1} \lambda^{3}\right)^{4}<6 \cdot 10^{18} C_{1}^{4} \lambda^{12} .
$$

This completes the proof of the main theorem.

REMARK 4.4. The first part of the above proof can be easily modified to show that a planar set $A$ having the $\varphi$-BLEP is relatively connected if

$$
\lim _{\varepsilon \rightarrow 0} \varphi(\varepsilon) \ln ^{2} \varepsilon=0 .
$$




\section{REFERENCES}

1. Adams, R. A., Calculus: A Complete Course, 6th Ed. Pearson Addison Wesley, 2006.

2. Alestalo, P., Trotsenko, D. A., Väisälä, J., Isometric approximation, Israel J. Math. 125 (2001), 61-82.

3. Alestalo, P., Trotsenko, D. A., Väisälä, J., Linear bilipschitz extension property, Sibirsk. Mat. Zh. 44 (2003), 1226-1238. Translation in Siberian Math. J. 44 (2003), 959-968.

4. Benyamini, Y., Lindenstrauss, J., Geometric Nonlinear Functional Analysis, Vol. 1, Amer. Math. Soc. Colloq. Publ. 48 (2000).

5. Matoušek, J., Lectures on Discrete Geometry, Grad. Texts in Math. 212 (2002).

6. Trotsenko, D. A., Väisälä, J., Upper sets and quasisymmetric maps, Ann. Acad. Sci. Fenn. Math. 24 (1999), 465-488.

7. Väisälä, J., Bilipschitz and quasisymmetric extension properties, Ann. Acad. Sci. Fenn. Math. 11 (1986), 239-274.

DEPARTMENT OF MATHEMATICS

PL 1100, HELSINKI UNIVERSITY OF TECHNOLOGY

FIN-02015 TKK FINLAND

E-mail: pekka.alestalo@tkk.fi
INSTITUT MATEMATIKI SO RAN

KOPTYUGA PROSPEKT 4

630090 NOVOSIBIRSK

RUSSIA

E-mail: trotsenk@math.nsc.ru 